\title{
SOME INEQUALITIES FOR MEANS DEFINED ON THE LORENTZ CONE
}

\author{
Yu-Lin Chang, Chien-Hao Huang, Jein-Shan Chen and Chu-Chin Hu
}

Abstract. In this paper, we define various means associated with Lorentz cones (also known as second-order cones), which are new concepts and natural extensions of traditional arithmetic mean, harmonic mean, and geometric mean, logarithmic mean. Based on these means defined on the Lorentz cone, some inequalities and trace inequalities are established.

Mathematics subject classification (2010): 26D07, 26B35.

Keywords and phrases: Mean, second-order cone, Lorentz cone, trace, SOC-convex, SOC-monotone.

\section{REFERENCES}

[1] T. Ando, Matrix Young inequalities, Operator Theory: Advances and Applications 75 (1995), 33-38.

[2] J. S. Aujla And H. L. Vasudeva, Convex and monotone operator functions, Annales Polonici Mathematici 62 (1995), 1-11.

[3] R. Bhatia, Matrix Analysis, Springer-Verlag, New York, 1997.

[4] R. Bhatia, Positive definite matrices, Princeton university press, 2005.

[5] R. BHATIA, Interpolating the arithmetic-geometric mean inequality and its operator version, Linear Algebra and Its Applications 413 (2006), 355-363.

[6] R. BHATIA AND C. DAVIS, More matrix forms of the arithmetic-geometric mean inequality, SIAM Journal on Matrix Analysis and Applications 14 (1993), 132-136.

[7] R. Bhatia AND F. KitTANeH, Notes on matrix arithmetic-geometric mean inequalities, Linear Algebra and Its Applications 308 (2000), 203-211.

[8] P. Bhatia And K.P. Parthas arathy, Positive definite functions and operator inequalities, Bulletin of the London Mathematical Society 32 (2000), 214-228.

[9] J.-C. BOURIN AND F. HIAI, Jensen and Minkowski inequalities for operator means and anti-norms, Linear Algebra and its Applications 456 (2014), 22-53

[10] P. S. Bullen, Handbook of Means and Their Inequalities, Mathematics and Its Applications 560, Kluwer Academic Publishers, 2003.

[11] B. C. CARLSON, Some inequalities for hypergeometric functions, Proceedings of the American Mathematical Society 17 (1966), 32-39.

[12] J.-S. CHEN, The convex and monotone functions associated with second-order cone, Optimization $\mathbf{5 5}$ (2006), 363-385.

[13] J.-S. ChEn, X. Chen, S.-H. PAN, AND J. ZHANG, Some characterizations for SOC-monotone and SOC-convex functions, Journal of Global Optimization 45 (2009), 259-279.

[14] J.-S. CHEN, X. CHEN, AND P. TSENG, Analysis of nonsmooth vector-valued functions associated with second-order cones, Mathematical Programming 101 (2004), 95-117.

[15] J. Faraut And A. KorÁnYi, Analysis on Symmetric Cones, Oxford Mathematical Monographs, New York: Oxford University Press, 1994.

[16] M. Fukushima, Z.-Q. LuO, And P. Tseng, Smoothing functions for second-order-cone complementarity problems, SIAM Journal on Optimization 12 (2002), 436-460.

[17] M. Hajua, P. S. Bullen, J. Matkows ki, E. Neuman, and S. Simic, Means and Their Inequalities, International Journal of Mathematics and Mathematical Sciences 20132013.

[18] R. A. Horn and C. R. Johnson, Matrix Analysis, Cambridge University Press, 1985.

[19] F. Kittaneh And M. KRnic, Refined Heinz operator inequalities, Linear Multilinear Algebra 61 (2013), 1148-1157. 
[20] A. KorÁnYI, Monotone functions on formally real Jordan algebras, Mathematische Annalen 269 (1984), 73-76.

[21] H. LeE AND Y. Lim, Metric and spectral geometric means on symmetric cones, Kyungpook Mathematical Journal 47, 1 (2007), 133-150.

[22] Y. LIM, Geometric means on symmetric cones, Archiv der Mathematik 75, 1 (2000), 39-45.

[23] Y. LiM, Applications of geometric means on symmetric cones, Mathematische Annalen 319, 3 (2001), $457-468$.

[24] E. Neuman, The weighted logarithmic mean, Journal of Mathematical Analysis and Applications, 188, 3 (1994), 885-900. 